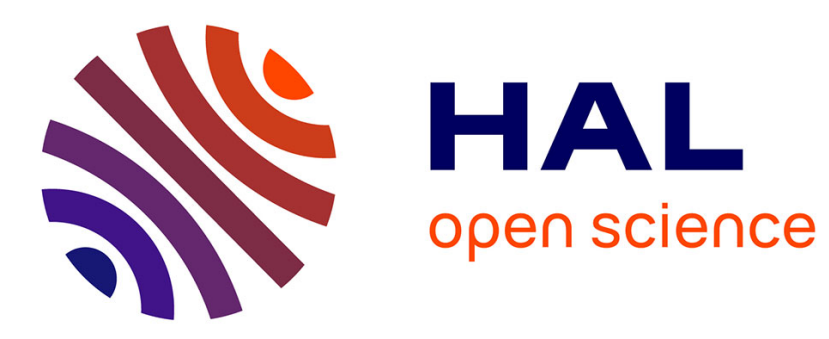

\title{
Konfliktlösung in Gemeinden unter muslimischer Herrschaft im mittelalterlichen Europa
}

\author{
Christian Müller
}

\section{To cite this version:}

Christian Müller. Konfliktlösung in Gemeinden unter muslimischer Herrschaft im mittelalterlichen Europa. Hrsg. David von Mayenburg,. Konfliktlösung im Mittelalter, 2, Springer, pp.355-361, 2021, Handbuch zur Geschichte der Konfliktlösung in Europa, 10.1007/978-3-662-56098-3_29 . halshs03322840

\section{HAL Id: halshs-03322840 \\ https://shs.hal.science/halshs-03322840}

Submitted on 19 Aug 2021

HAL is a multi-disciplinary open access archive for the deposit and dissemination of scientific research documents, whether they are published or not. The documents may come from teaching and research institutions in France or abroad, or from public or private research centers.
L'archive ouverte pluridisciplinaire HAL, est destinée au dépôt et à la diffusion de documents scientifiques de niveau recherche, publiés ou non, émanant des établissements d'enseignement et de recherche français ou étrangers, des laboratoires publics ou privés. 
„Konfliktlösung in Gemeinden unter muslimischer Herrschaft im mittelalterlichen Europa“.

In: Konfliktlösung im Mittelalter, Handbuch zur Geschichte der Konfliktlösung in Europa, Bd. 2. Hrsg. David von Mayenburg, Springer, Berlin Heidelberg, 2021, S. 355-361. https://doi.org/10.1007/978-3-662-56098-3_29

Christian Müller (IRHT - CNRS Paris)

\section{Zusammenfassung}

Muslimische Gemeinden entstanden im europäischen Mittelalter in Folge einer dauerhaften muslimischen Herrschaft, zunächst innerhalb der Führungsschicht, dann durch allmähliche Konversion autochthoner Bevölkerungskreise. Formen institutionalisierter Konfliktlösung stehen mit der Entwicklung des islamischen Juristenrechts und seiner Definition einer regulären Kadi-Gerichtsbarkeit sowie der Schlichtung (tahkim) in Verbindung. Der Kadi galt als vom Herrscher mit der Gerichtsbarkeit im Allgemeinen betraut, andere Richterämter (hākim, pl. hukkām) besaßen polizeiliche oder untersuchungsrichterliche Entscheidungsbefugnisse. Für islamische Territorien auf europäischem Boden sind Quellen- und Forschungslage sehr unterschiedlich. Der Eintrag behandelt die Gerichtsbarkeit im muslimischen Teil der Iberischen Halbinsel, al-Andalus, (711-1492) und im weniger gut dokumentierten Sizilien (827-1086), wohingegen für das islamische Malta (870-1090) keine Quellen zur Rechtsgeschichte erhalten sind.

\section{1. Überblick}

Muslimische Gemeinden entstanden im europäischen Mittelalter in Folge einer dauerhaften muslimischen Herrschaft, zunächst innerhalb der Führungsschicht, dann durch allmähliche Konversion autochthoner Bevölkerungskreise. Formen institutionalisierter Konfliktlösung stehen mit der Entwicklung des islamischen Juristenrechts und seiner Definition einer regulären Kadi-Gerichtsbarkeit sowie der Schlichtung (tahkim) in Verbindung. Der Kadi galt als vom Herrscher mit der Gerichtsbarkeit im Allgemeinen betraut, andere Richterämter (ḩākim, pl. hukkām) besaßen polizeiliche oder untersuchungsrichterliche Entscheidungsbefugnisse. Für islamische Territorien auf europäischem Boden sind die Quellen- und Forschungslage sehr unterschiedlich.

1) Die ab dem Jahre 711 eroberte Iberische Halbinsel (al-Andalus) blieb unter ihren umayyadischen Herrschern zunächst politisch von den ab 750 die islamische Welt regierenden Abbasiden unabhängig, weshalb einige Institutionen dort noch drei Jahrhunderte überdauerten. Der Anschluss an die allgemeine Entwicklung des Juristenrechts erfolgte jedoch bereits unter Hišām I. im Jahre 786-7 mit dem Wechsel zur mālikitischen Rechtsschule. Deren recht gut erforschte lokale Rechtstradition blieb bis zur Kapitulation Granadas 1492 trotz eines almohadischen Intermezzos im 12.-13. Jahrhundert dominant.

2) Dagegen war das zwischen 827 und 1086 in wechselnden Anteilen muslimisch beherrschte Sizilien von Anfang an eng mit der frühen mālikitischen Rechtstradition des nordafrikanischen Festlands verbunden. Quellen berichten von einigen sizilianischen Kadis, Juristen und Rechtsfällen, doch bleibt eine lokale Rechtstradition unerforscht. Diese wurde entweder später überdeckt oder war von Anfang an nicht markant genug, um heute noch greifbare Spuren 
eventueller Eigenheiten zu hinterlassen. Allerdings zeugen arabische Rechtsurkunden aus normannischer Zeit von islamischer Rechtspraxis auf Sizilien (Nef 2011, S. 570 ff.).

3) Aus dem zwischen 870-1090 unter islamischer Herrschaft stehenden Malta sind keinerlei Quellen zur Rechtsgeschichte erhalten, obwohl die auf einem arabischen Dialekt basierende Landessprache auf eine starke kulturelle Durchdringung verweist (Rossi 1987).

Unberücksichtigt bleibt im Folgenden die in anderen historischen Zusammenhängen stehende osmanische Herrschaft über Teile des Balkans ab Mitte des 14. Jahrhunderts.

\section{Gerichtliche Streitschlichtung}

Streitfälle (sing. hușūma) werden vor den Richter (Kadi, arab. qā dị) gebracht. Hierbei konnten Kläger ihre Ansprüche sowohl einseitig einklagen oder mit der Streitpartei vor Gericht erscheinen. Das Kadiamt als Institution islamischen Rechts entwickelte sich während des Untersuchungszeitraums trotz eines extremen Rechtskonservatismus gerade auch in Hinsicht seiner Bindung an Rechtsregeln und im Beweisrecht (Müller 2018). Das ab dem 9. Jahrhundert literarisch fassbare Juristenrecht beschreibt den Kadi-Gerichtsprozess und die Rollen der Prozessbeteiligten im Hinblick auf die Beweisgrundlagen eines Urteils (hukm). Erkennt der Beklagte den Anspruch an, kann der Richter ein entsprechendes Urteil fällen. Leugnet der Beklagte den Rechtsanspruch, obliegt dem Kläger der Beweis, zunächst durch Zeugen, die der Beklagte gegebenenfalls durch eine Gegenklage als befangen ablehnen kann. Gelingt dem Kläger der Zeugenbeweis nicht, kann der Beklagte seine ablehnende Position mittels Gerichtseid bekräftigen. Verweigert der Beklagte diesen Eid, erhält der Kläger daraufhin die Möglichkeit, seinen Rechtsanspruch per Eid zu bestätigen (Kap. 14. Dirks). Laut Prozessrecht konnten nur aufgrund von Zeugenbeweis, Anerkenntnis oder Eid festgestellte Tatsachen als Grundlage eines Kadi-Urteils dienen, Schriftstücke besaßen keinen Beweischarakter. Laut islamischem Beweisrecht musste der Zeuge muslimisch, frei und ehrbar sein, Frauen (Kap. 8. Hähnchen/Matzen) fungierten in einigen Bereichen als Zeuginnen, wobei zwei Frauen wie ein Mann zählten (Rechtsbücher des figh). In der Praxis wurde der Zugang zum Zeugenstatus ab dem 8. Jahrhundert eingeschränkt (Tyan 1960, S. 239-242), welcher sich zu beim Kadi akkreditierten Berufszeugen entwickelte. Sie wurden für die Beurkundungen von Rechtsgeschäften und Tatsachenbestätigungen bezahlt und bezeugten diese im Streitfall mündlich vor Gericht. Nicht alle Streitfälle wurden vor dem Richter aufgrund formalisierter Zeugenaussagen und per Urteil entschieden. Entscheidend war die Bezeugung auch geringwertiger Ansprüche jedoch im Erbfall, soweit Beteiligte selbst nicht mehr aussagen konnten. Unsere Quellen $\mathrm{zu}$ Gerichtsverfahren, Rechtsgutachten und Gerichtsurkunden protokollieren i.d.R. nicht den Verhandlungsverlauf, sondern geben eine Endbeurteilung wieder. Dennoch lassen sich anhand detaillierter Rechtsanfragen aus den Ahkām al-kubrā des Ibn Sahl (verstorben 1086) diverse gerichtliche Rollen und deren sich mit der Beweislage ändernde Zuweisung rekonstruieren (Müller 1999, S. 175-201).

\section{Kernprobleme der Forschung}

Hauptquellen zur Konfliktlösung in muslimischen Gemeinden sind systematische Rechtswerke und Responsa-Sammlungen (Fatwas) muslimischer Juristen. Während diese für al-Andalus reichlich vorhanden und ansatzweise erforscht sind (Lagardère 1995; Müller 1999; Fierro 2006; Calero 2000), erlaubt die spärliche Quellenlage bislang nicht, bei aus Sizilien stammenden 
Gelehrten eine vom nordafrikanischen Festland abweichende lokale Rechtstradition nachzuweisen (Muranyi 1997). Ein Kernproblem der Forschung zum islamischen Recht besteht im Verhältnis gerichtlicher Praxis zur juristischen Theorie (Müller 2016), welche etwa von Fadel (1997) für granadinische Rechtsgutachten des 14.-15. Jahrhunderts untersucht wurde. Einer dem Rechtsrealismus verpflichtete Erforschung der Kadi-Gerichtsbarkeit anhand detaillierter Fallanalysen setzt die Quellenlage mit einigen erhaltenen Notariatshandbüchern, Rechtskommentaren, Gutachten und wenigen Originalurkunden enge Grenzen. Während die meisten andalusischen Handbücher zur Marktaufsicht (hisba) sowie einige Notariatshandbücher in Übersetzung vorliegen, gilt dies für Rechtswerke nur ausnahmsweise (Qāḍī 'Iyāḍ 1998). Die Funktion des Kadi als Streitschlichter wirft mehrere Fragen auf: 1) die Regelgebundenheit richterlicher Entscheidungen, 2) beschränkte inquisitorische Befugnisse, 3) die Behandlung nicht „beweisbarer" Ansprüche sowie 4) das Verhältnis zu anderen Richterämtern in al-Andalus.

1) Entgegen des Weberschen Archetypus der Kadijustiz war der Kadi nicht nur dem Anspruch nach auch in al-Andalus stark regelgebunden. Hiervon zeugen neben den grundlegenden unveränderlichen Regelungen des Prozessrechts insbesondere die zahlreichen Schriften lokaler Juristen zu einzelnen Rechtsfragen (masā'il) und Rechtsgutachten (fatāwā). Die weitgehende Überschneidung identischer Lehrmeinungen in mehreren Rechtsfrage-Werken des 12. Jahrhunderts, etwa des Ša bī, Ibn Baštaġayr und Qāḍ̄i 'Iyāọ (Lirola Delgado 2004-17), verweist auf ihren normativen Charakter. Diese inhaltlich noch kaum ausgewerteten Werke zu Detailfragen der Rechtspraxis erfassten die gängigen Regelungen, ungeachtet dessen, ob sie selbst Teil der seit Jahrhunderten fixierten sakralen Rechtsdoktrin waren oder diese ergänzten. Insgesamt war der Kadi wohl stärker an Regeln des Juristenrechts gebunden, als dies das in der Forschung lange vorherrschende Paradigma eines inhärenten Theorie-Praxis Gegensatzes (Schacht 1964) vorsieht.

2) Die Kadi-Gerichtsbarkeit zeichnete sich juristisch dadurch aus, dass der Kadi nur ihm „offenkundigen“ Aspekte des Falles, Aussagen und Beweismittel der Streitparteien, bewertete, ohne selbst eine Untersuchung des Falles zur Aufdeckung verborgener Aspekte zu veranlassen. Dies führte etwa zur Ablehnung von Folter als Wahrheitsfindungsmittel im Kadi-Prozess (Johansen 1996). Damit war der Kadi in einem formalen Urteil (hukm) an die ihm vorliegenden „Beweise“ gebunden.

3) Angesichts des strikten Beweisrechts stellt sich die Frage, ob und wie der Kadi Klagen ohne Zeugenbeurkundungen handhabte, etwa wenn die Streitparteien diese nicht erhalten konnten oder aus Kostengründen vermieden. Eine bei unzureichender Beweislage durchaus auch vom Kadi praktizierte Möglichkeit bestand im Vergleich (șulh) zwischen den Streitparteien (Müller 1999, S. 212-215). Jenseits beweisbarer Rechtsansprüche boten Notariatshandbücher Vorlagen für Vergleiche bei Erbschaften und Legaten, bei der Bezahlung des verzögerten Teils der Brautgabe und der Begleichung anderer Schulden, sowie bei Totschlag und Körperverletzung, aber auch zur vorsorglichen Bezeugung eines beabsichtigten Rücktritts von einer Übereinkunft (Serrano 2016, S. 92 f.). Die Möglichkeit eines gleichwohl koranisch legitimierten Vergleichs wurde von andalusischen Juristen des 11. Jahrhundert kontrovers diskutiert, und zwar sowohl im Hinblick auf hiervon auszuschließende Rechtsansprüche, gesetzeswidrige Abmachungen, beweisrechtliche Voraussetzungen, als auch bei Verfügungen zulasten abhängiger Dritter. Eine umfassend sakralrechtlich fundierte Theorie des Vergleichs aus dem 13. Jahrhundert schloss die Übereinkunft von verbotenen Rechtsgeschäften explizit aus, da diese entgegen früherer 
Auffassungen das Ergebnis anfechtbar mache (Serrano 2016, S. 82 f., 85, 87-90). Angaben zum Verhältnis zwischen per Urteil, Schlichtung oder sonstiger ohne Urteilsentscheidung beendeter Kadi-Gerichtsfälle lassen sich aufgrund mangelnder Quellen vor den osmanischen Gerichtsregistern ab dem 15. Jahrhundert nicht machen. Der Vorteil eines formalen Urteils bestand in seiner Dauerhaftigkeit, da es nur durch ein Gegenurteil aufgehoben werden konnte. Angesichts der juristischen Vorbehalte gegenüber der Beweiskraft von Schriftstücken konnten die Kadis in der Frühphase Schriftstücke aus dem Archiv ihres Vorgängers zunächst nicht selbst als Urteilsgrundlage verwenden. Mit dem ab dem 10. Jahrhundert entwickelten ZweiPhasen-Konzept entsprach die Zeugensignatur auf einem Schriftstück dem „Auf-Sich-Nehmen“ einer späteren „Ableistung“ der Bezeugung, welche beliebig häufig und auch vor nachfolgenden Richtern durchgeführt werden konnte. Damit wurden die Urteilsurkunden des Kadi-Archivs ein Instrument zur langfristigen Wahrung subjektiver Rechte (Müller 2018).

4) Die erwähnte Beschränkung der Kadijustiz bei der Beweisermittlung wurde im umayyadisch geprägten al-Andalus durch mehrere Ämter aufgefangen, welche sowohl polizeiliche als auch richterliche Befugnisse besaßen. Der Marktaufseher (șāhib al-sūq) ist für Cordoba ab dem 8. Jahrhundert nachgewiesen (Chalmeta 1973). Die ehemals umayyadische Palastwache (šurța) mit polizeilichen Befugnissen wurde ab der ersten Hälfte des 9. Jahrhunderts nach Bevölkerungsgruppen in hohe und niedere Polizei getrennt, zu einer Zeit als Cordoba einen eigenen Stadtpräfekten (șāhib al-madīna) erhielt. Während des 10. Jahrhunderts existierte sogar eine Dreiteilung der Polizeigewalt. Im 11. Jahrhundert waren für den Kleinstaat Cordoba nunmehr der Stadtpräfekt, sowie der Marktvogt (șāhib al-šurța wal-sūq) bedeutsam. (Müller 1999, S. 107-128) Die „Beseitigung von Unrechtssituationen“ (mazālim) galt zunächst als Vorrecht des umayyadischen Emirs, der jenseits der ordentlichen Gerichtsbarkeit die Beschwerden seiner Untertanen aufgriff und auch die Mächtigen bestrafte. Im Zuge der Ausdifferenzierung des kalifalen Hofes wurde ab dem Jahre 937 eine eigene Petitionsgerichtsbarkeit an Wesire, später auch an einen juristisch geschulten Petitionsrichter (șāhib al-mazālim) zur technischen Prüfung der Beschwerden übertragen. (Müller 1999, S. 130133, 2010, S. 99 f.) Alle genannten Ämter konnten laut Formularbuch des im Jahre 1008 verstorbenen Ibn al-'Ațtāār (ediert 1983 und übersetzt 2000) als hākim (pl. hukkām) ein Urteil fällen, welches nach Zeugenbestätigung (išhād) rechtskräftig wurde (tanfíd al-hukm). Allerdings besaß nur der Kadi ein Archiv (dīwān al-qā dì ), in dem neben den Amtszeiten seiner Vorgänger seit dem 9. Jahrhundert auch die Kadi-Urteile registriert waren. Auch in Cordoba waren bestimmte Bereiche der Kadi-Gerichtsbarkeit vorbehalten (Müller 1999, S. 151, 162). Die verschiedenen, noch in umayyadischer Tradition stehenden außerordentlichen Gerichtsbarkeiten verschwinden unter den aus Nordafrika stammenden Herrscherdynastien der Almoraviden (1090-1142) und Almohaden (1160-1236). Neue Ämter ersetzten sie, etwa der dem Kadi nachgeordnete almoravidische șāhib al-ahkām (El Hour 2000-1). Während die Almoraviden eine strenge Anwendung der mālikitischen Rechtsdoktrin vertraten (Lagardère 1986, El Hour 2006, Fierro 1997, S. 459-465), verstanden sich die Almohaden als messianistische Reformbewegung und entwickelten eigene Rechtsansätze (Fierro 2005, 2016). Für die Marktaufsicht wurde damals die allgemein-islamische Bezeichnung muhtasib verwendet (Chalmeta 1973, S. 396 f., 472). Erst nach der almohadischen Niederlage und dem Verlust weiter Landesteile an christliche Herrscher findet sich im nunmehr nașridischen Granada (1232-1492) die Bezeichnung des „Marktamtes“ (hutțat al-sūq) (Calero Secall 2000, S. 413). Dort lebte das Kadiamt in der Verbindung mit der mālikitischen Rechtstradition bis zur Übergabe der Stadt an Kastilien weiter (Viguera 2000; Calero Secall 2000). Hiervon zeugen u. a. die zahlreichen 
arabischen Urkunden über Grundstücksverkäufe aus der Endzeit (Zomeño 2011). Neben der allgemeinen Gerichtsbarkeit des Kadi unterschieden die Juristen eine von den Streitparteien eingesetzte Schiedsgerichtsbarkeit (tahkim), welche als „spezielle Befugnis“ keine Ansprüche Dritter berühren dürfe, nicht strikt an Rechtsregeln gebunden war und bezüglich der Schlichtenden auch nicht den strengen formalen Anforderungen an einen Kadi entsprach. Juristischer Anknüpfungspunkt hierfür war die koranische Figur der „beiden Schlichter“ (hakamān) in Ehestreitigkeiten, welche aus den beiden Clans der Eheleute stammten (Fierro 2006). Die gleichzeitige Ernennung mehrerer Kadis in der Stadt Onda nach Zusammenbruch der Zentralgewalt im 11. Jahrhundert, womöglich eine verbreitete Praxis, diskutierte der Jurist alBāğī in Zusammenhang mit der Schiedsgerichtsbarkeit und verurteilte sie als bedauerliche Neuerung für das Kadiamt. (Serrano 2016, S. 77-83). Da Rechtsgutachten zumeist abstrakte Auffassungen vertreten, sind Anweisungen zur Schlichtung (tahkim) und Vergleich (șulh) selten bestimmten Ämtern zuzuordnen. Wie Serrano (2016) zeigt, versuchen die späteren Juristen, diese Formen ins System der Rechtsnormen einzubeziehen, wohingegen die Forschung die Schiedsgerichtsbarkeit früher Perioden zumeist außerhalb der Kadi-Gerichtsbarkeit ansiedelt (Tillier 2016).

Bibliographie

Calero Secall MI (2000) La justicia, cadíes y otros magistratos. In: Viguera Molíns MJ (Hrsg) El Reino nazarí de Granada (1232-1492). Politica, Instituciones, espacio y economía. Historia de España Menéndez Pidal, Bd VIII-3. Espasa Calpe, Madrid, S 367-427

Chalmeta Gendrón P (1973) El „señor del zoco“ en España. Edades media y moderna, contribución al estudio de la historia del mercado. Instituto Hispano-Arabe de Cultura, Madrid

Chalmeta Gendrón P (2010) El zoco medieval. Contribución al estudio de la historia del mercado. coll. Estudios Andalusíes 3. Fundación Ibn Tufayl de Estudios Árabes - Fundación Cajamar, Almeria

El Hour (2000-2001) Le șāḥib al-aḥkām à l'époque almoravide. In: Al-Andalus Maghreb: Estudios árabes e islámicos 8-9(1):49-64

El Hour R (2006) La Administración judicial almoravide en al-Andalus. Élites, negociaciones y enfrentamientos. Academia Scientarum Fennica, Helsinki

Fadel M (1997) Rules, Judicial Discretion, and the rule of law in Nașrid Granada. An analysis of al-Ḥadīqa al-mustaqilla al-naḍra fī al-fatāwā al-șādira 'an 'ulamā' al-ḥaḍra. In: Gleave R, Kermeli E (Hrsg) Islamic law. Theory and practice. I.B. Tauris, London, S 49-86

Fierro M (1997) La religion. Capitulo II. Derecho, teologia y heterodoxia. In: Viguera Molíns MJ (Hrsg) El retroceso territorial de al-Andalus. Almorávides y Almohades siglos XI al XIII. Historia de Espana Ramón Menéndez Pidal, Bd VIII-2. Espasa Calpe, Madrid, S 459-481

Fierro M (2005) Doctrina y práctica juridicas bajo los almohades. In: Cressier P, Fierro M, Molina L (Hrsg) Los Almohades. Problemas y perspectivas. Bd 2. CSIC, Madrid, S 895-935

Fierro M (2006) Ill-treated women seeking divorce. The Qur'ānic two arbiters and judicial practice among the Mālikis in al-Andalus and North Africa. In: Masud MK, Peters R, Powers D (Hrsg) Dispensing Justice in Islam. Qadis and their Judgments. Brill, Leiden, S 232-247 
Fierro M (2016) Legal doctrine and practice under the Almohads. In: Fierro M (Hrsg) The Almohadic revolution. Politics and religion in the Islamic West during the twelfth-thirteenth centuries. 2. Aufl. Routledge, Milton Park/New York, part VIII, S 1-34 (Übersetzung von Fierro 2005)

Ibn 'Abdūn (1981) Sevilla a comienzos del siglo XII. El tratado de Ibn 'Abdún, übers. Garcia Gomez E und Lévy-Provencal E. Servicio de Publicaciones del Ayuntamiento de Sevilla, Sevilla

Ibn al-'Aț̣āar (1983) Formulario notarial hispano-árabe por el alfaquí y notario cordobés Ibn al-'Ațtār (s.X) (Kitāb al-watā'iq wal-siğillāt). Textedition Chalmeta P, Corriente F, Academia Matritense del Notariado, Instituto Hipano-Árabe de Cultura, Madrid

Ibn al-'Ațtāā (2000) Formulario Notarial y judicial andalusí. Übersetzt von Marugán M, Einleitung Chalmeta P, Fundación Matritense del Notario, Madrid

Johansen B (1996) Vérité et torture. Ius commune et droit musulman entre le Xe et le XIIIe siècle. In: Séminaire de Françoise Héritier (Hrsg) De la violence. O. Jacob, Paris, S 123-168

Lagardère V (1986) La haute judicature à l'époque almoravide en al-Andalus. In: Al-Qanțara 7:135-228

Lagardère V (1995) Histoire et Société en Occident Musulman au Moyen Âge. Analyse du Míyār d'alWanšarīsī. Consejo Superior de Investigationes Cientificas, Madrid

Lirola Delgado J (Hrsg) (2004-17) Biblioteca de al-Andalus. 10 Bände. Fundación Ibn Tufayl de Estudios Árabes, Almeria

Müller C (1999) Gerichtspraxis im Stadtstaat Córdoba. Zum Recht der Gesellschaft in einer mālikitischislamischen Rechtstradition des 5./11. Jahrhunderts. Brill, Leiden

Müller C (2010) Redressing injustice. Mazāalim jurisdictions at the Umayyad Court of Córdoba. In: Fuess A, Hartung J-P (Hrsg) Court cultures in the muslim world. Seventh to nineteenth centuries. Routledge, London, S 93-104

Müller C (2016) Recht I. Vormodern. In: Brunner R (Hrsg) Islam. Einheit und Vielfalt einer Weltreligion. Kohlhammer, Stuttgart, S 237-257

Müller C (2018) The power of the pen. Cadis and their archives. From writings to registering proof of a previous action taken. In: Bausi A et al (Hrsg) Manuscripts and archives. Comparative views on record-keeping. de Gruyter, Berlin, S 361-385

Muranyi M (1997) Beiträge zur Geschichte der Hadīt und Rechtsgelehrsamkeit der Mālikiyya in Nordafrika bis zum 5. Jh. d. H. Bio-bibliographische Notizen aus der Moscheebibliothek von Qairawān. Harrassowitz, Wiesbaden

Nef A (2011) Conquérir et gouverner la Sicile islamique au XIe et XIIe siècles. École Française de Rome, Rom

Qāḍī 'Iyāọ (1998) Muḥammad b. 'Iyāḍ (m. 575/1179). Maḍāhib al-ḥukkām fī nawāzil al-aḥkām ( La actuation de los jueces en los procesos judiciales). Traducción y estudio Delfina Serrano, CSIC, Madrid

Rossi E (1987) Malta. In: Encyclopaedia of Islam. New Edition VI:295a-b 
Schacht J (1964) An introduction to Islamic law. Clarendon Press, Oxford

Serrano D (2016) Bringing arbitration (tahkim) and conciliation (sulh) under the qāậ $\vec{s}$ purview in Mālikī al-Andalus (10th to 12th centuries C.E.). In: Tillier M (Hrsg) Arbitrage et conciliation dans l'Islam médiéval et moderne. Presses Universitaires de Provence, Aix-en-Provence, S 73-99

Tillier M (2016) Arbitrage et conciliation aux premiers siècles de l'Islam. Théories, pratiques et usages sociaux. In: Tillier M (Hrsg) Arbitrage et conciliation dans l'Islam médiéval et moderne. Presses Universitaires de Provence, Aix-en-Provence, S 27-52

Tyan É (1960) Histoire de l'organisation judiciaire en pays d'Islam. Brill, Leiden

Viguera M (2000) La religión y el derecho. In: Viguera Molíns MJ (Hrsg) El Reino nazarí de Granada (1232-1492). Politica, Instituciones, espacio y economía. Historia de España Menéndez Pidal, Bd VIII-4. Espasa Calpe, Madrid, S 175-190

Zomeño A (2011) From private collections to archives. How christians kept arabic legal documents in Granada. In: Al-Qantara 32:461-479 